\title{
System Design for Implementing Distributed Modular Architecture to Reliable Surgical Robotic System
}

\author{
Eisuke Aoki ${ }^{1}$, Takashi Suzuki $^{1}$, Etsuko Kobayashi ${ }^{1}$, Nobuhiko Hata ${ }^{1}$, \\ Takeyoshi Dohi $^{2}$, Makoto Hashizume ${ }^{3}$, and Ichiro Sakuma ${ }^{1}$ \\ 1 Institute of Environmental Studies, Graduate School of Frontier Sciences, The \\ University of Tokyo \\ http://bme.pe.u-tokyo.ac.jp/index_e.html \\ \{aoki, t-suzuki, etsuko, sakuma\}@miki.pe.u-tokyo.ac.jp \\ 2 Graduate School of Information Science and Technology, The University of Tokyo \\ noby@atre.t.u-tokyo.ac.jp \\ dohi@miki.pe.u-tokyo.ac.jp \\ 3 Department of Disaster and Emergency Medicine, Kyushu University \\ mhashi@dem.med.kyushu-u.ac.jp
}

\begin{abstract}
A method that resolves the two competing requirements for a surgical robotic system (reliability and scalability) is discussed, along with its preliminary implementation in a master-slave system. The proposed method enables an architecture that can be scaled without impairing the performance of the surgical robotic system. Our method uses an optimized architecture consisting of two components: a common object request broker architecture (CORBA) and a master-slave system that typically operates using two-way communication links between a client and a remote server (the dedicated system architecture). In this new architecture, the surgical robotic system can maintain a reliable performance and can integrate with various systems in a transparent manner, regardless of the hardware, operating system, or programming language. Our method was evaluated by recording all the available surgical information, and shows a reliable scalability for a surgical robotic system requiring real-time operation, regardless of the condition of the components of a CORBA-based system.
\end{abstract}

\section{Introduction}

Many telerobotic systems using distributed modular architectures have been developed over the last few years. The advantages of using network-based systems built on top of distributed computing systems technology are the reduction in system costs, the arbitrary location of clients, dynamic access to remote expertise as required, and the decreased costs of operator training. Following the current trends in modern distributed system design, open reconfigurable and scalable architectures can be built using standard middleware software for distributed object computing. As one of several standard middleware software packages available, many studies using surgical robotic systems have been reported 
employing the common object request broker architecture (CORBA) package [12. CORBA uses an Object Request Broker (ORB) as the middleware that establishes a client/server relationship between objects. The client can invoke a method on the server object across a network in a transparent manner without knowing where the application servers are located, or what programming language and operating system are being used. In addition, the components of a CORBA-based system can be implemented and run independently to implement the application, and can be easily integrated into new systems. Schorr et al. reported on the application of a CORBA system to control an imageguided surgical robot, where they controlled an MRI-compatible biopsy robot by sending the control information using CORBA 34. However, there have been several reports that CORBA is not always an effective, flexible, and robust technique for Internet-based robotics systems. One of the technological problems associated with CORBA is how to guarantee stable and reliable control under interference from a CORBA system. Generally, real-time software is required to control mechatronic systems, such as robots, and to develop a stable and reliable control system under limited computational resources, it is important to evaluate the overall system load imposed on the computer system. However, in the distributed modular architecture of a computer aided surgical system, where the system configuration may change depending on the clinical requirements, it is difficult to estimate the system load before the design of the system. In recent years, a master-slave manipulator system has been applied to lessinvasive laparoscopic surgery [5]. In contrast to an image-guided control system, the master-slave manipulator system is a relatively slow imaging system, and it requires a higher control frequency. On the other hand, to realize safe and accurate surgical operation, the integration of a surgical navigation system employing a master-slave surgical manipulator is necessary, and for this purpose, a distributed modular architecture is desirable. Thus, it is important to design a control system that meets these two competing requirements, and we set out to resolve these two competing requirements (scalability and reliability) in our surgical robotic system. This paper describes the following system components.

1. The use of an optimized architecture consisting of two components: a dedicated system architecture and CORBA, and

2. The development of an intermediate system to resolve any interference occurring between two architectures.

The above allow a surgical robotic system to maintain a reliable performance and to integrate various systems in a transparent manner, regardless of the hardware, operating system, or programming language used.

\section{System Architecture}

\subsection{System Overview}

It is important for a system to have sufficient computational power to handle the necessary information during processing. However, even if a system has sufficient performance, in general, it cannot handle situations that occur at random. 
Non real time system (CORBA)

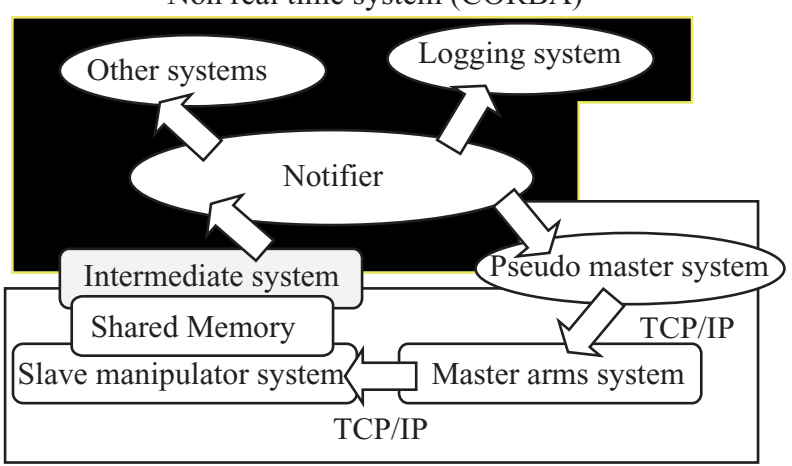

Real time system (Dedicated system architecture)

Fig. 1. System architecture

A system needs to function stably under such circumstances, and for the stable transmission of data using a CORBA-based system, the synchronous transmission of data is desirable for reliable communication among distributed objects. It is very difficult to define priorities for multiple objects connected to each other, unless the real time performance of the subsystems is well defined. In the case of a computer-aided surgery system, the configuration of the system may change, depending on the clinical requirements of specific cases. In the design of our prototype system, we prepared an intermediate system that allowed adjustments between the real time master-slave manipulator system and the CORBA-based system to be made. The system we developed consisted of a control system designed for real time control of the surgical master-slave manipulator system, a CORBA-based system that realized a distributed modular architecture, and an intermediate software system that connected these two systems(Fig. 1).

\subsection{A Master-Slave Manipulator System with a Dedicated Communication System}

The master-slave manipulator system consisted of a pair of master arms (MASTER) and a slave manipulator system (SLAVE). In the MASTER system(Fig. 3), an operator sits in front of a monitor showing a laparoscopic view and controls the master manipulators, using footswitches to command the SLAVE. The SLAVE system uses three slave robotic manipulators with seven degrees of freedom that include a grasping function, and an endoscope with a variable viewing angle [6.7]. The slave robotic manipulators are located on passive positioning arms for easier presetting of the manipulators before surgical operation. The position and orientation of the manipulators and the endoscope were measured using a Polaris optical positioning sensor (Northern Digital Inc., http://www.ndigital.com). The motion of the master arms was recognized as 

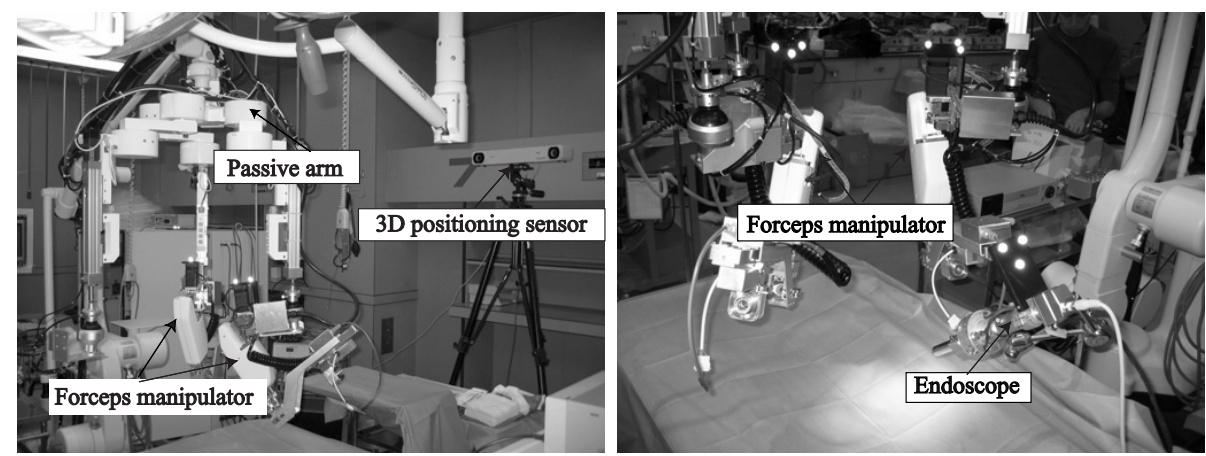

Fig. 2. Surgical slave manipulator system

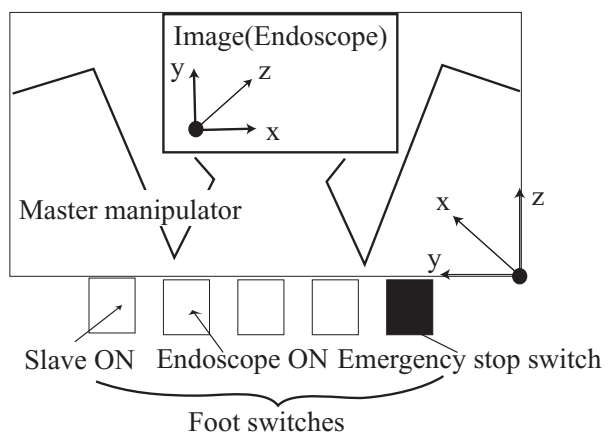

Fig. 3. Master arms system

the motion commands of the endoscope's coordinates, and this motion was transformed to the manipulator's coordinates. The required computation for the SLAVE control was conducted using a real time base. The MASTER and SLAVE were connected using a dedicated communication channel employing TCP/IP protocol. These two systems were tightly connected as a single client device and a server robot. As an alternative to the MASTER, a pseudo-MASTER could be used that could store the time series data from the master arms' motion and related control information, and could relay this data to the SLAVE. The pseudo-MASTER was also connected with the SLAVE using a dedicated communication channel, and in addition, this system was connected to the other distributed objects using CORBA. Using this pseudo-MASTER, the SLAVE could accept motion commands from the other objects using CORBA.

\subsection{Common Object Request Broker Architecture (CORBA)}

CORBA defines a framework for developing distributed applications. As many implementations support different operating systems, we chose the Adaptic Com- 
munication Environment (ACE) and the Object Request Broker (TAO) developed by Washington University, USA [8]. The developed system uses a CORBAbased system to interface with the distributed objects in the system. As an example of a distributed object that could be connected to the master-slave manipulator system, we implemented a logging system that recorded time-series data for the following control information:

- Commands from the master arm system to the slave manipulator system: the position and orientation of the master-manipulators and foot switches (On and Off).

- The status of the slave manipulators: position and orientation of the slave manipulators.

- The viewing direction of the endoscope.

- The set position of the slave manipulators: The set position of each passive arm holding the slave manipulator (the three slave manipulators, endoscope, and Polaris 3-D positioning sensor).

\subsection{Intermediate System}

We prepared an intermediate system located between the master-slave manipulator system and the communication system using CORBA software. The intermediate system receives data from the master-slave manipulator system in an asynchronous manner by means of a shared memory. It then sends the data to the other objects using CORBA in a synchronous manner to make the communication reliable. Introduction of this intermediate system loosely connected the master-slave manipulator system and the communication system using CORBA software. It prevented any interference with the robot control system due to instantaneous decrease of the system performance caused by communication overload and communication errors. Using this intermediate system, we were able to maintain the performance of the master-slave manipulator system and ensure the reliability of the entire system while keeping connectivity to the communication system based on CORBA. The developed system is shown in Figure 4 To evaluate the system stability under operating conditions, the proposed system design was subjected to the following tests using the experimental conditions shown in Figure 4

- Logging the system using the proposed system design.

- Carrying out the master-slave experiment using a communication rate of 100 msec.

- Using the total recorded surgical information for evaluation.

\section{Results and Discussion}

We implemented the system described in Section 2, shown in Figure 4 and we conducted experiments in operating the master-slave surgical manipulator 


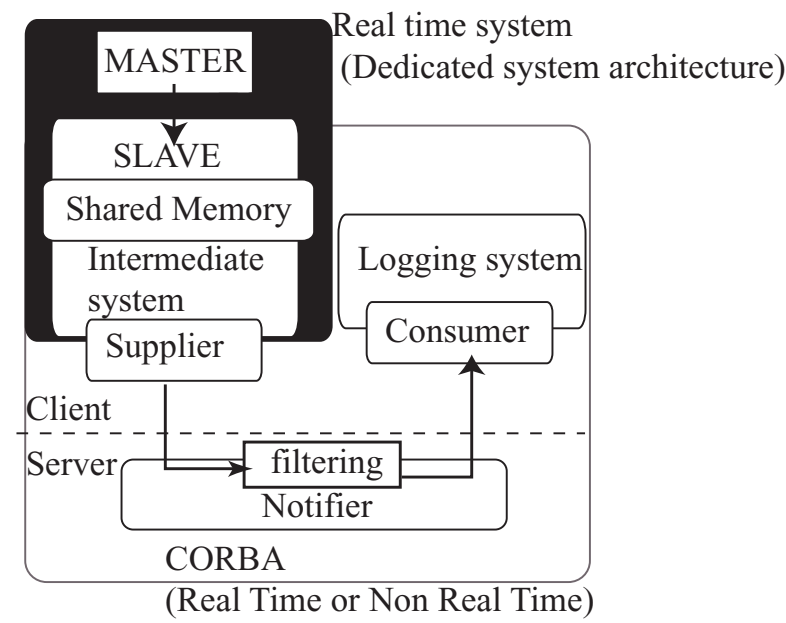

Fig. 4. System architecture in experiment

system. We also recorded the time-series data from the control data of the manipulators using CORBA in the logging system. We could successfully operate the master-slave manipulator and were able to record the control data without any problems. Figure 5 shows examples of the recorded data from the manipulator motion. Figures 5(a) and 5 b) show the position of the slave manipulator. The manipulator was only activated when the foot switch was activated. The data shows the expected behavior of the slave manipulator. Figure 5)(c) shows the change in joint angle during operation. We also tested the system without the intermediate system connecting the communication system based on CORBA and the real time control system for the master-slave manipulator in place. Synchronous transmission was used in these experiments to maintain reliable communication. Under these conditions, the real time control system for the manipulator suffered from the influence of coexisting processes of the CORBA communication system, resulting in an unstable system operation. By reinserting the intermediate system, we could successfully interact with the communication processes of CORBA with real time control of the manipulator. The data stored in the logging system was also transferred to the pseudo-master system, and used to repeat the same recorded motions of the manipulator. The slave manipulator repeated the same motions in a stable manner. In the application of the system in a computer aided surgery environment, distributed objects in the system will not always be in a real time system. If we adopt a system with close connections that require a strict real time operation, such as manipulator control, then these non-real time objects can form a bottleneck that will determine the overall system performance. Schorr et al. reported the application of a CORBA-based system to control an image guided surgical robot. They controlled an MRI-compatible biopsy robot by sending its control information 
(a) Foot switch

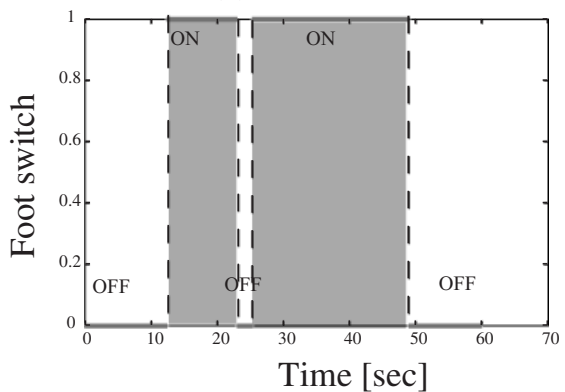

(b) Position of slave manipulator

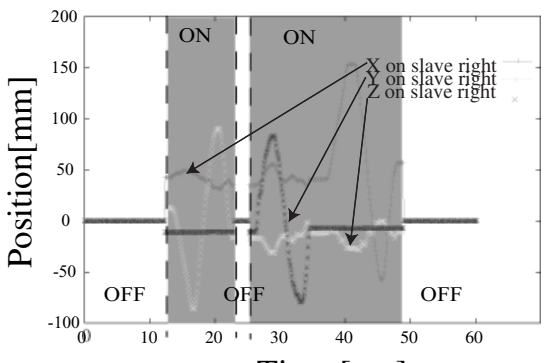

Time $[\mathrm{sec}]$

(c) Joint angle of slave manipulator

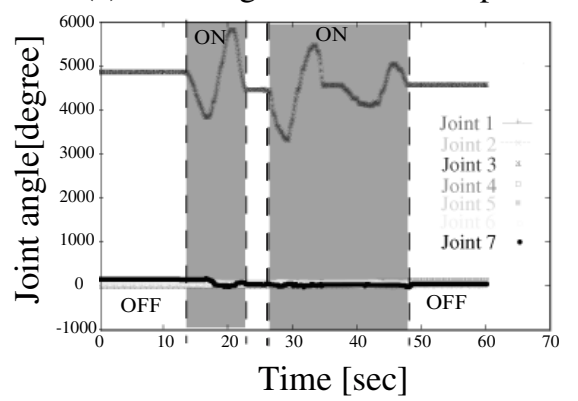

Fig. 5. Log data of logging system;(a)Foot switch, (b)Position of the slave manipulator, (c)Each joint angle of slave manipulator

using CORBA 34. Considering the nature of an MRI scanner, it seems that the required bandwidth for communicating navigation data in that case is not as high as the bandwidth requirements for communicating to our master-arms and slave-manipulators or for the dynamic motion control of our robotic manipulators. Thus, it is effective to utilize CORBA as the communication interface. When we combine the master-slave manipulator system for less invasive surgery, or for a surgical navigation system or surgical simulation system, then in general, the required transmission frequency of the navigation data is not so high. On the other hand, the scalability of a system that allows for the connection of various types of intraoperative imaging devices and instrumentation with positional data is more important. From this consideration, we propose the use of the intermediate system that will compensate for differences in the required real time performance among the distributed objects, while maintaining a reliable and extendable data communication at relatively low frequency using CORBA.

\section{Conclusion}

We have proposed a method to resolve the two competing requirements of a surgical robotic system: scalability and reliability, and we have shown the following: 
- Our system design combined real time objects, such as a master-slave manipulator control system with other non-real time objects used in a computeraided surgical assistance system. With the proposed system design, the reliability and stability of real time systems and scalability of a CORBA-based system were realized at the same time.

- We developed an intermediate system with real time and asynchronous processing to compensate for the real time and non-real time nature of the distributed objects. This system enabled the coexistence of the dedicated system architectures required for real time processing and CORBA.

- As an example of this function, we developed a logging system for the motion control data from the manipulators and a pseudo master system for the manipulator operation. We confirmed the stable real time control of the surgical master-slave manipulator together with data communication to the other distributed objects.

In future work, we will incorporate a surgical navigation system using the masterslave surgical robotic manipulator to realize safe and accurate surgical operation of this system.

\section{References}

1. SONGMIN JIA and KUNIKATSU TAKASE. Internet-based robotic system using corba as communication architecture. Journal of Intelligent and Robotic Systems, 34:121-134, 2002.

2. A.Pernozzoli, C.Burghart, J.Brief, S.Habfeld, J.Raczkowsky, J.Muhling, U.Rembold, and H.Worn. A Real-time CORBA Based System Architecture for Robot Assisted Craniofacial Surgery. In Medicine Meets Virtual Reality 2000 J.D. Westwood et al. (Eds.) IOS Press, 2000, 2000.

3. A. Bzostek, R.Kumar, N.Hata, O. Schorr, R. Kikinis, and R. H .Taylor. Distributed Modular Computar-Integrated Surgical Robotic Systems:Implementation using modular software and network systems. In proc, MICCAI '00, pages 969-978, 2000.

4. Oliver Schorr, Nobuhiko Hata, Andrew Bzostek, Rajesh Kumar, Catherina Burghart, Russel H. Taylor, and Ron Kikinis. Distributed Modular ComputarIntegrated Surgical Robotic Systems:Architecture for Intelligent Object Distribution. In proc, MICCAI'00, pages 979-987, 2000.

5. G.H.Ballantyne. Robotic surgery,telerobotic surgery,telepresence, and telementoring. In Springer-Verlag New York Inc., pages 1389-1402, 2002.

6. Takashi Suzuki, Eisuke Aoki, Etsuko Kobayashi, Takayuki Tsuji, Kozo Konishi, Makoto Hashizume, and Ichiro Sakuma. Development of forceps manipulator for assisting laparoscopic surgery. In proc of CARS, page 1338, 2004.

7. Takemasa Hashimoto, Etsuko Kobayashi, Ichiro Sakuma, Kazuhiko Shinohara, Makoto Hashizume, and Takeyoshi Dohi. Development of wide-angle view laparoscope using wedge prisms. Journal of Robotics and Mechatronics, pages 129-137, 2004.

8. David Levine and Sumedh Mungee. The design and performance of real-time object request brokers. Computer Communications, 21, 1998. 